\title{
Psychiatrische und psychotherapeutische Behandlung als Ersatzmaßnahme nach Art. 237 StPO in der Schweiz
}

\author{
Voraussetzungen und Grenzen aus forensisch-psychiatrischer Perspektive
}

Irina Franke ${ }^{1}$ Tobias Vogel $^{1} \cdot$ Marc Graf $^{1}$

Eingegangen: 2. Juli 2015 / Angenommen: 8. Januar 2016 / Online publiziert: 25. Januar 2016

(C) Die Autor(en) 2016. Dieser Artikel ist auf Springerlink.com mit Open Access verfügbar.

\begin{abstract}
Zusammenfassung Die Schweizerische Strafprozessordnung sieht die Möglichkeit der Anordnung von psychiatrischer und psychotherapeutischer Behandlung als Auflage zur Entlassung aus der Untersuchungshaft vor („Ersatzmaßnahmen“). Im Gegensatz zu den gesetzlichen Regelungen für die gerichtliche Anordnung von ambulanten und stationären Maßnahmenbehandlungen im Schweizerischen Strafrecht (entsprechend dem deutschen Maßregelvollzug) gibt es für die Anordnung von psychiatrisch-psychotherapeutischer Behandlung als Ersatzmaßnahme keine verbindliche Regelung. Eine sachverständige Begutachtung ist nicht zwingend. Durch die besondere Situation einer Therapieverpflichtung vor Bestehen eines rechtskräftigen Urteils können sich diverse psychotherapeutische, ethische und juristische Problemstellungen ergeben. Dazu gehören Aspekte der Diagnose- und Indikationsstellung, die mögliche präjudizielle Wirkung einer solchen Anordnung, die NutzenRisiko-Abwägung einer daraus resultierenden Behandlung und die Frage der Kostenübernahme. Eine Auswertung über den Zeitraum von 2 Jahren von 9 durchgeführten Ersatzmaßnahmen in der Forensischen Ambulanz für Erwachsene in Basel zeigte, dass nicht alle Fälle vorher begutachtet werden, dass nach der Anordnung einer solchen Maßnahme bis zur Gerichtsverhandlung durchschnittlich fast ein Jahr (338 Tage) vergeht und im Urteil nicht immer eine gerichtliche Weisung zur Fortsetzung der Behandlung angeordnet wird. Ersatzmaßnahmen können in bestimmten Fällen sinnvolle Alternativen darstellen, die Anordnung
\end{abstract}

Irina Franke

Irina.Franke@upkbs.ch

1 Forensisch-Psychiatrische Klinik, Universitäre Psychiatrische Kliniken Basel, Wilhelm Klein Str. 27, 4012 Basel, Schweiz sollte sich aus Sicht der Autoren immer auf eine sachverständige Begutachtung stützen.

Schlüsselwörter Schweizerische Strafprozessordnung · Ersatzmaßnahmen - Ambulante forensisch-psychiatrische Behandlung $\cdot$ Begutachtung

\section{Psychiatric and psychotherapeutic treatment as alternative measures according to Art. 237 of the Swiss code of criminal procedure}

Prerequisites and boundaries from forensic psychiatric perspectives

\begin{abstract}
The Swiss code of criminal procedure provides the option of psychiatric and/or psychotherapeutic treatment orders as a prerequisite for releasing a person from investigative custody ("alternative measures"). In contrast to the legal regulations for inpatient and outpatient treatment court orders in criminal law (equivalent to the German forensic commitment), the Swiss code of criminal procedure does not provide mandatory requirements for psychiatric and psychotherapeutic treatment as an alternative. Expert opinions are not obligatory. Due to the special situation of involuntary treatment prior to passing a final verdict, several psychotherapeutic, ethical and legal problems can emerge. These include aspects of the diagnosis and indications, the potentially prejudicial effect of such a treatment order, weighing the risk-benefit ratio of the treatment resulting from this and the question of who is responsible for bearing the treatment costs. An evaluation of nine treatment orders over a period of 2 years in the forensic outpatient department for adults at the Basel Psychiatric University Hospital showed that after issuing
\end{abstract}


such an order and beginning treatment, the mean duration until the court proceedings was nearly 1 year (338 days), an expert opinion was not a standard procedure and the court did not confirm the treatment order to be continued in every case. From the authors' perspective such treatment orders are a useful alternative in specific cases but should always be referred for a psychiatric expert opinion.

Keywords Swiss code of criminal procedure Alternative measures · Forensic psychiatric outpatient treatment . Expert opinion

\section{Einleitung}

Die Kernaufgaben forensischer Psychiatrie sind die Begutachtung von juristisch relevanten Auswirkungen psychischer Störungen und die Behandlung von straffällig gewordenen psychisch erkrankten Personen. Die Disziplin steht dabei auch in der Schweiz wegen verschiedener Besonderheiten unter besonderer öffentlicher Beachtung: Bei der gutachterlichen Tätigkeit stehen Qualitätssicherung und Einfluss des Sachverständigengutachtens auf das Gerichtsurteil im Zentrum der Diskussionen [1-3]. Bei der Behandlung psychisch kranker Straftäter stellen u. a. die gesellschaftlichen Zweifel an deren Wirksamkeit sowie die Abwägung zwischen Eingriff in die Persönlichkeitsrechte und öffentlichen Sicherheitsinteressen Herausforderungen dar. Daraus ergibt sich aus psychiatrischer Sicht u. a. die Notwendigkeit, die Möglichkeiten und Grenzen der Beeinflussbarkeit delinquenten Verhaltens durch therapeutische Maßnahmen kritisch zu reflektieren. Dies gilt insbesondere für Behandlungen, die von Beginn an im ambulanten Rahmen durchgeführt werden.

Bei Behandlungen im Rahmen strafrechtlich angeordneter gerichtlicher Maßnahmen (in der Schweiz: Art. 56 ff. StGB) kann der forensische Psychiater/Psychotherapeut seine Behandlung auf eine gerichtliche Tat- und Schuldfeststellung und eine gutachterliche Risikobeurteilung stützen, die neben der Begründung des Eingriffs in die Persönlichkeitsrechte eine wesentliche Grundlage zur Planung therapeutischer Interventionen darstellt. In der Praxis werden psychiatrische Behandlungen aber auch vor Abschluss des Gerichtsverfahrens im Rahmen von Ersatzmaßnahmen bei der Entlassung aus Untersuchungs- oder Sicherheitshaft, im vorgezogenen Vollzug oder durch Strafbefehle angeordnet. Psychiatrische und psychotherapeutische Behandlung auf unfreiwilliger Basis ohne vorherige gerichtliche Klärung der Tat- und Schuldfrage wirft verschiedene Fragen auf, die in diesem Beitrag diskutiert werden.

\section{Rechtliche Grundlagen}

Die seit 2011 in der Schweiz kantonsübergreifend einheitlich geregelte Strafprozessordnung [4] sieht in Art. 237 vor, dass das zuständige Gericht unter dem Gebot der Verhältnismäßigkeit anstelle von Untersuchungs- oder Sicherheitshaft auf Antrag eine oder mehrere mildere Maßnahmen anordnen kann, wenn diese den gleichen Zweck wie die Haft erfüllen. Folgende Maßnahmen können angeordnet werden:

- Sicherheitsleistung (Kaution),

- Ausweis- und Schriftensperre,

- Auflage, sich nur oder nicht an einem bestimmten Ort oder in einem bestimmten Haus aufzuhalten,

- Auflage, sich regelmäßig bei einer Amtsstelle zu melden,

- Auflage, einer geregelten Arbeit nachzugehen,

- Kontaktverbot zu bestimmten Personen und

- Auflage, sich einer ärztlichen Behandlung oder einer Kontrolle zu unterziehen.

Als Voraussetzungen für Untersuchungs- und Sicherheitshaft gelten, dass die beschuldigte Person eines Verbrechens oder Vergehens dringend verdächtig ist und ernsthaft $\mathrm{zu}$ befürchten ist, dass sie (Art. $221 \mathrm{StPO}$ ):

- sich durch Flucht dem Strafverfahren oder der zu erwartenden Sanktionen entzieht (Fluchtgefahr);

- Personen beeinflusst oder auf Beweismittel einwirkt, umso die Wahrheitsfindung zu beeinträchtigen (Verdunklungsgefahr);

- durch schwere Verbrechen oder Vergehen die Sicherheit anderer erheblich gefährdet, nachdem sie bereits früher gleichartige Straftaten verübt hat (Fortsetzungsgefahr) oder

- ihre Drohung, ein schweres Verbrechen auszuführen, wahrmachen wird (Ausführungsgefahr).

Die rechtliche Funktion von psychiatrisch-psychotherapeutischer Behandlung als Ersatzmaßnahme ist es somit zu verhindern, dass sich die Haftgründe der Fortsetzungs- und Ausführungsgefahr verwirklichen. Dies beinhaltet wie bei anderen gerichtlich angeordneten Maßnahmen den Aspekt der Verhinderung weiterer Delikte. In der Strafprozessordnung ist nicht festgelegt, welche Voraussetzungen für die Anordnung der Ersatzmaßnahme, sich einer ärztlichen Behandlung oder Kontrolle zu unterziehen, zu erfüllen sind. Damit besteht eine erhebliche Diskrepanz zum Strafrecht, das dezidierte Voraussetzungen für die Anordnung therapeutischer Maßnahmen formuliert, wenngleich die angeordneten bzw. durchgeführten therapeutischen Interventionen in beiden Fällen zumindest für den ambulanten Behandlungsrahmen vergleichbar sind.

Nach Schweizerischem Strafrecht [5] müssen zur gerichtlichen Anordnung einer therapeutischen Maß- 
nahme nach Art. 56 a) eine Gefahr für weitere Straftaten bestehen, der mit Strafe allein nicht zu begegnen ist, b) ein Behandlungsbedürfnis des Täters vorliegen oder die öffentliche Sicherheit dies erfordern und c) die Voraussetzungen der Art. 59-61, 63 oder 64 erfüllt sein. Zudem darf der mit der Maßnahme verbundene Eingriff in die Persönlichkeitsrechte des Täters im Hinblick auf die Wahrscheinlichkeit und Schwere weiterer Straftaten nicht unverhältnismäßig sein (Art. 56 Abs. 2 StGB). Der psychiatrische Sachverständige hat sich zu äußern über a) die Notwendigkeit und die Erfolgsaussichten einer Behandlung des Täters, b) die Art und die Wahrscheinlichkeit weiterer möglicher Straftaten und c) die Möglichkeiten des Vollzugs der Maßnahme (Art. 56 Abs. 3 StGB). Insbesondere ist den einzelnen Maßnahmeartikeln (Art. 59-63 StGB) als Bedingung vorausgesetzt, dass eine schwere psychische Störung des Täters vorliegt. Das Schweizerische Strafrecht kennt neben den stationären forensischen Behandlungsmaßnahmen (Art. 59-61 StGB) auch die sogenannte ambulante Maßnahme (Art. 63 StGB), die mit der Möglichkeit einer maximal 2-monatigen stationären Einleitung von Beginn an ambulant geführt wird. Bei der Anordnung von Ersatzmaßnahmen vor Entlassung aus der Untersuchungshaft ist eine psychiatrische Begutachtung hingegen nicht obligatorisch. Eine solche Begutachtung erfolgt dennoch in den meisten Fällen, wobei sich der Sachverständige oft vor der definitiven Fertigstellung des Gutachtens in Form einer sogenannten Vorabstellungnahme zur Anordnung von Ersatzmaßnahmen äußern soll.

\section{Psychiatrisch-psychotherapeutische Aspekte}

Gemäß schweizerischer und internationaler Rechtsprechung sowie gängiger Lehre [6] ist jeder ärztliche Heileingriff, selbst dann, wenn er lege artis erfolgt, eine Verletzung der Persönlichkeit des Patienten. Die wichtigste Voraussetzung für die Rechtmäßigkeit des Heileingriffs ist die rechtswirksame Einwilligung; das Schweizerische Zivilgesetzbuch (ZGB Art. 28 Abs. 2; [7]) nennt darüber hinaus abschließend überwiegendes privates oder öffentliches Interesse oder Rechtfertigung durch ein Gesetz. Auch psychiatrische und psychotherapeutische Maßnahmen sind ärztliche Heileingriffe in diesem Sinne.

Psychiatrische Behandlung beinhaltet ein weitreichendes Spektrum an Interventionen, die sich aus dem Konzept eines biopsychosozialen Entstehungsmodells psychischer Erkrankungen ableiten. Sie kann medikamentöse Behandlung, Psychoedukation, sozialpsychiatrische Betreuung, Einbezug des Umfelds, tagesstrukturierende Beschäftigung, Abstinenzkontrollen und Fallkoordination (Case Management) umfassen. Je nach Krankheitsbild und individuellem Funktionsniveau des
Patienten werden diese Maßnahmen unterschiedlich stark im Behandlungsplan gewichtet. Die Indikation für diese Behandlungen wird bei allen schweren, teils chronifiziert verlaufenden psychiatrischen Erkrankungen gestellt (z. B. Schizophrenien, bipolare Störungen, Depressionen, hirnorganische Störungen/Demenzen; [8-11]). Diese Behandlungen sind nicht delikt-, sondern störungsspezifisch und nicht in jedem Fall auf Heilung ausgerichtet. Wenn keine anhaltende Remission erreicht werden kann, zielt die Therapie auf weitgehende Verbesserung der Lebensqualität des Betroffenen (z. B. Symptommanagement) ab. Im forensisch-psychiatrischen Kontext ist das bei den oben genannten Krankheitsbildern und bei einem engen Zusammenhang zwischen psychiatrischer (Akut-)Symptomatik und Delinquenz ausreichend rückfallpräventiv.

Die Indikationsstellung für eine Psychotherapie setzt voraus, dass eine behandlungsbedürftige und mit psychotherapeutischen Mitteln behandelbare Erkrankung vorliegt, dass die zu behandelnde Person urteilsfähig ist und über die vorgesehene Behandlung aufgeklärt wird. Darüber hinaus sollte sie über die persönlichen, motivationalen und intellektuellen Fähigkeiten verfügen, die Therapieinhalte auffassen und umsetzen zu können. Bei manchen Störungsbildern kann die Entwicklung einer Behandlungsbzw. Veränderungsmotivation explizit auch Teil der ersten Behandlungsphase sein, sodass fehlende Motivation per se keinen primären Ausschlussgrund für eine psychotherapeutische Behandlung darstellen muss.

$\mathrm{Zu}$ beachten ist ferner, dass psychotherapeutische Maßnahmen wie alle ärztlichen Interventionen unerwünschte Wirkungen haben, erfolglos bleiben oder fehlerhaft durchgeführt werden können. Während die allgemeine Psychotherapieforschung sich diesem Thema inzwischen auch durch wissenschaftliche Untersuchungen genähert hat und von einer Rate unerwünschter Psychotherapiewirkungen zwischen 3 und $15 \%$ ausgeht [12], gibt es für die forensische Psychotherapie keine entsprechenden Untersuchungen. Der Stellenwert einer sorgfältigen Indikationsstellung und Qualitätskontrolle ist im Bereich der forensischen Psychotherapie zusätzlich von besonderer Bedeutung, da eine direkte Verbindung zum Rückfallrisiko für Delinquenz besteht.

ImerweitertenSinnekönnenalleBehandlungsmaßnahmen der forensischen Psychiatrie als deliktorientiert gelten, da die Indikation hierfür nach Erstellung einer individuellen Delikthypothese unter deliktpräventiven Gesichtspunkten gestellt wird. Deliktorientierte Psychotherapie im engeren Sinne bedeutet v. a. die fundierte Auseinandersetzung mit delinquenzauslösenden oder -aufrechterhaltenden Faktoren der Persönlichkeit bzw. Beziehungsgestaltung mit dem Ziel, eine Minderung des individuellen Rückfallrisikos zu erreichen [13]. Eine wesentliche Voraussetzung zur Durchführung einer solchen Behandlung ist neben dem Vorliegen einer durch psychotherapeutische Methoden behandelbaren 
psychischen Erkrankung die gerichtliche Tat- und Schuldfeststellung. Nur auf dieser Basis können eine individuelle Delikthypothese, Risikofaktoren und Behandlungsziele erarbeitet werden.

In der forensischen Therapie sind diese grundsätzlichen Überlegungen durch den kombinierten Auftrag von (Heil-)Behandlung und öffentlichem Interesse bzw. Risikoreduktion tangiert. Das bezieht sich z. B. auf das Recht auf Vertraulichkeit und Datenschutz, das gegenüber der anordnenden Behörde aufgelockert ist [14]. Die Freiwilligkeit einer Psychotherapie ist im Zwangskontext ebenfalls zu hinterfragen, da sich eine Ablehnung in den meisten Fällen nicht günstig auf die weitere Vollzugsplanung auswirken wird. Diese Aspekte mittelbaren Zwangs sind auch bei Psychotherapien wirksam, die als Ersatzmaßnahmen angeordnet werden, allerdings ohne dass die Täterschaft der betroffenen Person gerichtlich festgestellt wurde. Daraus ergeben sich in der Praxis verschiedene Schwierigkeiten:

Beschuldigte/Patienten geben gegenüber Gutachtern und/oder Verfahrensbeteiligten oft an, eine hohe Therapiemotivation zu haben, die oft v. a. durch die Aussicht auf Entlassung aus der Haft zustande kommt, sich später aber wenig nachhaltig zeigt. Der Beginn einer Psychotherapie als Ersatzmaßnahme ist für die inhaftierte Person eine Möglichkeit, die Untersuchungshaft zu verkürzen, die sie durch eine bloße Zustimmung verwirklichen kann. Die Motivation zur Psychotherapie begründet sich hier also nicht auf Einsicht in das Vorliegen problematischer Persönlichkeitsanteile bzw. psychischer Störungen, sondern auf der Abwendung eines als aversiv empfundenen Zustands, was im Vergleich $\mathrm{zu}$ anderen denkbaren Motivatoren als außergewöhnlich starker Faktor zu gewichten ist. Als weiterer Faktor ist zu berücksichtigen, dass Personen, die in diesem Kontext zur Therapie zugewiesen werden, häufig nicht wissen, was Psychotherapie bedeutet. Für den Therapeuten heißt das, dass ein erheblicher Teil der Anfangsarbeit darin besteht zu versuchen, ein motivationales Gegengewicht zu entwickeln, um überhaupt in die eigentliche therapeutische Arbeit einsteigen zu können. Auch die diagnostische Beurteilung ist unter diesen Voraussetzungen erschwert. Die Durchführbarkeit einer Ersatzmaßnahme lässt sich vor allen Dingen zu Beginn nur an formalen Kriterien (Termineinhaltung, Terminhäufigkeit) messen und dem Auftraggeber kommunizieren. Damit kann ein sehr technisches Bild von psychotherapeutischen Prozessen entstehen, in dem fälschlicherweise der Eindruck vermittelt wird, dass ein inhaltlich nicht näher definiertes Gespräch mit einer psychotherapeutisch ausgebildeten Fachperson als Therapie qualifiziert und geeignet ist, Symptome und problematisches Verhalten zu reduzieren bzw. neues Verhalten aufzubauen.

Eine weitere Schwierigkeit in der (deliktorientierten) therapeutischen Arbeit im Rahmen von Ersatzmaßnahmen stellt die Situation des nichtabgeschlossenen Strafverfahrens dar. Das führt dazu, dass der Beschuldigte/Patient einerseits aufgefordert ist, in der Therapie transparent über deliktrelevante Verhaltensweisen oder Symptome zu berichten, damit ein sinnvolles Risikomanagement möglich ist bzw. eine „motivierte Teilnahme“ an der Therapie bestätigt werden kann, und andererseits sein strafprozessuales Grundrecht, sich nicht selbst belasten zu müssen, gefährdet sein kann. Dies ist insbesondere relevant, solange die Tatvorwürfe strittig sind oder wenn die Therapieindikation durch psychiatrische Laien gestellt wird. Ersatzmaßnahmen können so im Einzelfall präjudizielle Wirkung entfalten, indem die Einhaltung der Weisung zur Therapie im Zirkelschluss als Grund für ihre Notwendigkeit und implizit als Schuldanerkennung angesehen wird. Aus Sicht der Autoren kann eine deliktorientierte Behandlung unter diesen Voraussetzungen nicht lege artis erfolgen.

Unbedingte Voraussetzung für die Anordnung solcher Maßnahmen sollte ein Sachverständigengutachten sein, das das Vorliegen einer psychiatrischen Diagnose, die ambulant mit Mitteln der Psychiatrie bzw. Psychotherapie behandelt werden kann, bestätigt. Diese Diagnose, ihr Bezug zur vorgeworfenen Delinquenz, und der Zeitraum, in dem bei optimalem Verlauf eine (deliktrelevante) Besserung eintreten kann, sollten aus psychiatrischer Sicht bei der Anordnung von Ersatzmaßnahmen berücksichtigt werden. In Fällen, in denen beispielsweise der krankheitswertige schädliche Gebrauch einer psychotropen Substanz eine maßgebliche Rolle bei der Delinquenzentstehung spielte, kann durch Abstinenzkontrollen vergleichsweise rasch günstig auf das Risikoprofil eingewirkt werden. Anders verhält es sich z. B. bei Delinquenz im Zuge von persönlichkeitsstrukturellen Störungen oder Störungen der sexuellen Präferenz, bei denen therapeutische Interventionen längerfristig angelegt sind. Vor allem unmittelbar nach Entlassung aus der Untersuchungshaft, d. h. bei Beginn der Behandlung, kann (noch) nicht von einer tragfähigen therapeutischen Beziehung ausgegangen werden. Die Entwicklung einer solchen Beziehung ist vor dem Hintergrund des laufenden Strafverfahrens und der Doppelfunktion des Therapeuten als Behandler und Berichterstatter ohnehin herausfordernd. Gleichzeitig bestehen bei diesen Störungen im ambulanten Behandlungsrahmen keine Möglichkeiten der objektiven Kontrolle von Risikofaktoren, und eine kurzfristige Verbesserung des Risikoprofils durch psychotherapeutische Maßnahmen ist bei solchen Konstellationen nicht $\mathrm{zu}$ erwarten. Diese unterschiedlichen zeitlichen Behandlungsprofile müssen dem Rechtsanwender bei der Anordnung von Ersatzmaßnahmen gegenwärtig sein.

Ersatzmaßnahmen werden zeitlich befristet angeordnet. In der Praxis werden die Abstände der Kontrolle (z. B. durch Verlaufsberichte) und die Berücksichtigung von fachärztlichen Empfehlungen zur Modifikation der Behandlung unterschiedlich gehandhabt. Ersatzmaßnahmen, die über mehrere 
Jahre dauern, können unter dem Einfluss des laufenden Strafverfahrens auf die Transparenz in der Therapie mittelbar therapieschädigende Auswirkungen haben. Diese Beeinträchtigung der Therapiequalität ist $\mathrm{u}$. $\mathrm{U}$. auch nach der gerichtlichen Schuldfeststellung nicht mehr aufzuarbeiten. Andererseits sollte die Dauer der Ersatzmaßnahme vor Gericht auch im Hinblick auf die Anordnung weiterführender strafrechtlicher Maßnahmen berücksichtigt werden: Abhängig von Störungsbild und Behandlungsverlauf ist die Anordnung einer ambulanten Maßnahme nach einer 2-jährigen Therapie im Rahmen einer Ersatzmaßnahme im Einzelfall möglicherweise unnötig. Auf der anderen Seite liegen bei Personen, die im Zusammenhang mit einer psychischen Erkrankung straffällig werden, häufig Diagnosen vor, die die Motivation, freiwillig eine Behandlung aufzusuchen, einschränken können. Hier haben Ersatzmaßnahmen möglicherweise positive Effekte, v. a., wenn es sich um Störungsbilder handelt, bei denen eine möglichst schnelle Behandlung mit einer besseren Behandlungsprognose korreliert.

Ungeklärt ist oft auch die Frage nach der Kostenübernahme für solche Behandlungen. Die gängige Praxis ist, dass die Maßnahme als ambulante Behandlung über die Krankenversicherung abgerechnet wird. Dies ist beim Vorliegen einer psychischen Störung unproblematisch. Schwieriger gestaltet sich die Kostenfrage, wenn sich der Verdacht auf eine psychische Störung nicht bestätigt, wenn z. B. eine Paraphilie ohne psychiatrische Komorbidität und Leidensdruck vorliegt, oder bei Diagnosen aus dem Bereich der „Z-Diagnosen“ des ICD-10. Dies kann v. a. im Verhältnis zur Dauer mancher Ersatzmaßnahmen problematisch sein.

Wissenschaftliche Untersuchungen über die Wirksamkeit von psychiatrischer bzw. psychotherapeutischer Behandlung als Ersatzmaßnahme gibt es nach Kenntnis der Autoren nicht. Dabei stellt sich neben dem Einfluss der Behandlungen auf die Legalprognose v. a. die Frage, ob es sich bei einer eventuellen, noch nachzuweisenden Prognoseverbesserung um einen spezifischen Effekt psychotherapeutischer Interventionen handeln würde oder um das Zusammenwirken unspezifischer Wirkfaktoren, wie den Kontrollaspekt durch die regelmäßigen Termine, die konstante Konfrontation mit den Straftatvorwürfen und nachwirkende Strafsensibilität.

\section{Daten}

In der forensischen Ambulanz für Erwachsene in Basel befanden sich zwischen 01.01.2013 und 31.12.2014 neun männliche Personen im Rahmen von Ersatzmaßnahmen in psychiatrisch-psychotherapeutischer Behandlung. In die Auswertung wurden alle Fälle einbezogen, die sich am 01.01.2013 in Behandlung befanden, die vor 31.12.2014 die Behandlung abgeschlossen haben oder sich am
31.12.2014 noch in Behandlung befanden. Bei den noch nicht abgeschlossenen Verfahren wurde der 31.12.2014 als Stichtag zur Berechnung der bisherigen Dauer der Maßnahme verwendet. Von 9 Fällen wurden 6 vor Anordnung der Ersatzmaßnahme psychiatrisch begutachtet und mit einer Ausnahme eine Empfehlung für Psychotherapie ausgesprochen. In den 3 Fällen, in denen die Anordnung ohne Begutachtung erfolgte, ging es um den Straftatvorwurf der sexuellen Handlungen mit einem Kind, und keiner der Betroffenen wies eine relevante psychiatrische Anamnese auf. Eine detaillierte juristische Begründung über die allgemeine Formulierung hinaus, dass eine ambulante psychiatrische/psychotherapeutische/deliktorientierte Behandlung geeignet ist, um der Wiederholungsgefahr zu begegnen, erfolgte in diesen Fällen nicht.

Es zeigte sich eine durchschnittliche Dauer der Ersatzmaßnahme von 338 Tagen bis zum erstinstanzlichen Urteil. Von den 5 juristisch abgeschlossenen Fällen wurden nur in 2 Fällen gerichtlich weiterführende therapeutische Maßnahmen angeordnet (in einem Fall eine ambulante therapeutische Maßnahme nach Art. $63 \mathrm{StGB}$, in dem zweiten Fall eine Weisung nach Art. 44 Abs. 2 StGB). Bei den Fällen, in denen das Gericht später keine therapeutische Maßnahme anordnete, bestätigten sich entweder die Straftatvorwürfe nicht; das Gericht ging davon aus, dass die begonnene Therapie auch auf freiwilliger Basis fortgesetzt werden würde, oder folgte der Einschätzung der Behandler, dass eine Psychotherapie nicht durchführbar ist. Diagnostisch lagen bei den analysierten Fällen Pädophilie $(n=4)$, Persönlichkeitsstörungen mit Substanzabusus $(n=2)$ und Abhängigkeit $(n=1)$ vor, bei 2 Personen konnte keine Diagnose nach ICD10 gestellt werden. Die Behandlungen erfolgten störungsund, sofern möglich, deliktspezifisch. Bei den Personen mit der Diagnose einer Persönlichkeitsstörung konnte keine Psychotherapie durchgeführt werden (Terminversäumnisse, Substanzkonsum). Bei den Personen mit der Diagnose einer Pädophilie zeigten sich unterschiedliche Verläufe, aus unserer Sicht v. a. abhängig davon, inwieweit die Straftatvorwürfe bereits im Ermittlungsverfahren eingeräumt wurden. In den Fällen, in denen das Gericht eine weiterführende Therapie anordnete, wurde diese ohne Unterbrechung beim bisherigen Therapeuten weitergeführt (Tab. 1).

\section{Diskussion und Schlussfolgerungen}

Ersatzmaßnahmen sind ein Instrument der Schweizerischen Strafprozessordnung, das die Anordnung psychiatrischer und psychotherapeutischer Behandlungen vor dem Abschluss des Strafverfahrens ermöglicht. Die Auswertung der Fälle in Basel zeigt, dass diese Maßnahme durchschnittlich fast ein Jahr dauerte, bevor das erstinstanzliche Urteil gefällt wurde, und das Gericht nur in der Minderheit der 
Tab. 1 Durchgeführte Behandlungen im Rahmen von Ersatzmaßnahmen in der Forensischen Ambulanz Basel vom 01.01.2013 bis zum 31.12.2014

$\begin{array}{llll}\text { Behandlungen } & N=\mathbf{9}(\mathbf{m}) & & \\ \text { Davon begutachtet } & \mathbf{6} & & \\ \text { Verfahrensstand } & 5 & & \\ \text { - Abgeschlossen } & 4 & & \\ \text { - Laufend } & & & \text { Gerichtsurteil } \\ \text { Dauer der Ersatzmaßnahme [Tage; Mittelwert (Range) } & \mathbf{3 3 8}(124-673) & & 1 \text { (Weisung) } \\ \text { - Abgeschlossene Verfahren }(n=5) & \mathbf{2 6 5}(9-713) & \text { Strafbefehl } & 3 \\ \text { - Laufende Verfahren }(n=4) & \text { Eingestellt } & 0 & 0 \\ \text { Verfahrensstand/Tatvorwürfe } & 0 & 0 & 1 \text { (Art. } 63 \text { StGB) } \\ \text { Sexuelle Handlungen mit Kindern }(n=4) & 1 & 1 \text { (keine Therapie) } & 1 \\ \text { Illegale Pornographie im Internet }(n=1) & 0 & 0 & 1 \text { (keine Therapie) } \\ \text { Häusliche Gewalt/Stalking }(n=3) & 0 & 0 \\ \text { Eigentumsdelinquenz (Betrug; } n=1) & & \end{array}$

$m=$ männlich.

Fälle eine Fortführung der Therapie im Rahmen einer strafrechtlichen Maßnahme anordnet.

Psychiatrische und psychotherapeutische Interventionen sollten als Ersatzmaßnahme nur angeordnet werden, wenn die Indikation hierfür durch einen Sachverständigen sorgfältig gestellt wird. Wenn die Anordnung als Automatismus erfolgt, besteht die Gefahr, dass die forensische Psychiatrie Funktionen der Strafverfolgungsbehörden übernimmt, die sie nicht erfüllen kann bzw. sollte: Bei den oben beschriebenen Basler Fällen entstand teilweise der Eindruck, dass die Weisung zur psychiatrisch-psychotherapeutischen Behandlung besonders bei Straftatvorwürfen zur Anwendung kam, bei denen eine Verlängerung der Untersuchungshaft aus Verhältnismäßigkeitsgründen nicht mehr möglich war und bei denen gleichzeitig ein hohes Maß an Unsicherheit bezüglich der Ausführungs- bzw. Rückfallgefahr bestand (Stalking, Drohungen, pädosexuelle Delikte). Damit verbunden ist auch die Frage nach den Kosten, die mit Beginn der Ersatzmaßnahme über die Abrechnung zulasten der Krankenkasse nicht mehr als Kosten der Strafverfolgung in Erscheinung treten. In diesen Situationen ist eine genaue Beurteilung erforderlich, ob ambulante psychiatrische Maßnahmen tatsächlich geeignet sind, der Problematik zu begegnen. Bei einer Überschätzung des Risikos für weitere Delinquenz erfolgt eine Therapie auf unfreiwilliger Basis mit diversen potenziellen Nebenwirkungen (Therapieschäden, Intransparenz). Bei einer Überschätzung der rückfallpräventiven Wirksamkeit einer Ersatzmaßnahme besteht durch den wenig kontrollierenden Rahmen das Risiko erneuter Delinquenz, die möglicherweise länger unentdeckt bleibt. Für Betroffene ist die Aussicht auf Haftentlassung nachvollziehbar immer der größtmögliche motivationale Faktor. Bei den analysierten Fällen wurde von allen Betroffenen beim Erstgespräch sinngemäß angegeben, sie hätten der Behandlung zugestimmt, um aus der Haft entlassen zu werden. Der Aufbau einer alternativen motivationalen Grundlage ist nach unseren Erfahrungen in Einzelfällen möglich, aber langwierig. Die
Tab. 2 Voraussetzungen

Tatvorwürfe weitgehend unstrittig

Fachärztlich festgestellte Diagnose einer behandelbaren psychischen Erkrankung und kausaler Zusammenhang zwischen festgestellter psychischer Erkrankung und Delinquenz

Allgemeine Voraussetzungen für die Durchführung einer Psychotherapie sind erfüllt

Kurzfristige Risikominimierung durch Therapie ist nicht primäres Ziel

Keine weiteren delinquenzbegünstigenden Faktoren

Vorwiegend Kontrollfunktion (z. B. bei Abstinenzkontrollen) oder Verabreichung von Medikamenten (z. B. Depotmedikation bei Personen mit Erkrankungen aus dem schizophrenen Formenkreis) Limitierte Dauer und regelmäßige Überprüfung, ggf. rasche Modifikation

Bereitschaft vonseiten der Strafverfolgungsbehörden, bei Risikoverhalten Sicherheitshaft anzuordnen

adäquate Prüfung der Therapiemotivation bzw. -indikation ist unter dieser Voraussetzung eine besondere Herausforderung, die einem forensisch erfahrenen Psychiater vorbehalten bleiben sollte. Problematisch erscheint angesichts der analysierten Fälle aber auch, dass seitens der Sachverständigen eine Therapieempfehlung gegeben wird, deren Verhältnismäßigkeit bei der Anordnung von Ersatzmaßnahmen offenbar anders beurteilt wird als bei der späteren gerichtlichen Beurteilung des Sachverhalts; hier wird die Therapieauflage in weniger der Hälfte aller Fälle aufrechterhalten. Andererseits besteht die Möglichkeit, dass Personen, die ohne äußeren Faktor trotz vorhandener Indikation nie eine Therapie besucht hätten, durch eine Ersatzmaßnahme eine Behandlung aufnehmen, die im besten Fall Leidensdruck und Legalprognose positiv beeinflusst. Um diese Aspekte genauer zu analysieren, wäre eine gesamtschweizerische Evaluation von Fällen mit psychiatrisch-psychotherapeutischen Ersatzmaßnahmen nach Entlassung aus der Untersuchungshaft sinnvoll. Die aus Sicht der Autoren erforderlichen Voraussetzungen für eine legalprognostisch wirksame Therapie im Rahmen einer Ersatzmaßnahme sind in Tab. 2 zusammengefasst. 


\section{Einhaltung ethischer Richtlinien}

Interessenkonflikt I. Franke, T. Vogel und M. Graf erklären, dass kein Interessenkonflikt besteht.

Dieser Beitrag beinhaltet keine Studien an Menschen oder Tieren.

Open Access Dieser Artikel unterliegt den Bedingungen der Creative Commons Attribution License. Dadurch sind die Nutzung, Verteilung und Reproduktion erlaubt, sofern der/die Originalautor/en und die Quelle angegeben sind.

\section{Literatur}

1. Hachtel H, Aenis L, Sahin D, Graf M (2014) Der Einfluss des Sachverständigengutachtens auf das Urteil aus psychiatrischer und juristischer Sicht. Schweiz Z Kriminol (2):3-9

2. Heer M (2011) Art. 183 StPO. Schweizerische Strafprozessordnung (StPO), Jugenstrafprozessordnung. Basler Kommentar. Helbing Lichtenhahn Verlag, Basel

3. Stratenwerth G, Kuhlen L (2004) Strafrecht. Allgemeiner Teil, 5. neu bearb. Aufl. ed. Academia iuris. Carl Heymanns Verlag, Köln, $424 \mathrm{~S}$

4. Schweizerische Strafprozessordnung. 05.10.2007 [23.02.2015]. http://www.admin.ch/opc/de/classified-compilation/20052319/ index.html
5. Schweizerisches Strafgesetzbuch. http://www.admin.ch/opc/de/ classified-compilation/19370083/index.html

6. Bär W, Honsell H, (1994) Handbuch des Arztrechts. Schulthess Polygraphischer Verlag, Zürich, XXXV, $469 \mathrm{~S}$

7. Schweizerisches Zivilgesetzbuch (ZGB). [cited 2015 24.02.]. http://www.admin.ch/opc/de/classified-compilation/19070042/ index.html\#a80

8. Gaebel W (2005) Behandlungsleitlinie Schizophrenie, Bd 1. Springer Science \& Business Media, Berlin

9. Bauer M (2013) S3-Leitlinie-Diagnostik und Therapie bipolarer Störungen. Springer-Verlag, Berlin

10. Müller U et al (2003) Nationale und internationale Demenz-Leitlinien im Vergleich. Fortschr Neurol Psychiatr 71:285-295

11. Bauer $M$ et al (2004) Biologische Behandlung unipolarer depressiver Störungen: Behandungsleitlinien der World Federation of Societies of Biological Psychiatry (WFSBP). Wissenschaftliche Verlagsgesellschaft Stuttgart, Stuttgart

12. Haupt M-L, Linden M, Strauss B (2013) Definition und Klassifikation von Psychotherapie-Nebenwirkungen. In: Strauss B, Linden M (Hrsg) Risiken und Nebenwirkungen von Psychotherapie. Medizinische Wissenschaftliche Verlagsgesellschaft, Berlin

13. Hoffmann K (2005) Grundlagen der forensischen Psychotherapie. Psych Pflege 11(06):333-342

14. (FMH), V.d.S.Ä.u. Ä. Behandlung von Patientinnen und Patienten. http://www.fmh.ch/files/pdf11/Patientenbehandlung_D1.pdf 